\title{
Is virtual reality the future of undergraduate medical teaching in the socially distanced learning environment?
}

\author{
Authors: Francesca Holden, ${ }^{\mathrm{A}}$ Irem Arican, ${ }^{\mathrm{A}}$ Elizabeth Heitz ${ }^{\mathrm{A}}$ and Christopher Bell ${ }^{\mathrm{A}}$
}

\section{Introduction}

In the era of COVID-19, the introduction of novel teaching methods to support socially distanced learning will provide a new key element in developing medical education. Virtual reality (VR) is an innovative teaching method which can be used in a variety of clinical settings to enhance learning. The objective of this teaching study was to compare whether teaching using VR was more effective than traditional teaching methods in undergraduate medical students. The second objective was to assess whether VR was an appropriate teaching method for elderly care topics.

\section{Materials and methods}

The experimental teaching study was conducted between September 2019 and February 2020. Twenty-nine undergraduate medical students were divided into two arms. One received VR teaching on 'home environment visits' and traditional teaching (an MS PowerPoint presentation) on 'mobility' (group A; $n=15$ ). The second arm received VR teaching on 'mobility' and traditional teaching on 'home environment visits' (group B; $n=14$ ). Students were invited to complete a pre-and post-teaching questionnaire. The results of both questionnaires were analysed and compared. Pivot tables were used to aggregate the answers generating results as a total sum per question. The total sum in the answer of each question was compared between the 'pre-teaching' and 'postteaching' questionnaires, enabling a percentage change to be calculated.

\section{Results and discussion}

Group A (VR teaching on 'home environment visits') demonstrated a $47 \%$ increase in the ability to assess hazards in the home environment. Group B (traditional teaching on "home environment visits') demonstrated a $6 \%$ increase. Group A demonstrated a $47 \%$ increase in the number of hazards they were able to name in the home environment, group B demonstrated a $27 \%$ increase. Group A (traditional teaching on 'mobility') demonstrated a $36 \%$ increase in confidence with aiding a patient to stand with a walking aid. Group B (VR teaching on 'mobility') demonstrated an $85 \%$ increase. Furthermore, 93\% (27/29) students volunteered positive feedback as free text comments, highlighting VR teaching as innovative and memorable. Currently there are limited reports in the literature of VR being used for undergraduate student learning. It is particularly useful in environments which are difficult to simulate in real life, or where usual teaching methods are disrupted due to social distancing. The results from this study show that the use of VR to teach medical students elderly care topics produced better learning compared with more traditional methods. Limitations include the high cost of VR video production, which may reduce as VR teaching becomes more prevalent. Another limitation of this study is the inclusion of students across a variety of year groups, possibly introducing varying degrees of baseline knowledge.

\section{Conclusion}

This pilot study demonstrated that there is a role for VR in teaching medical students on elderly care topics. The positive outcomes of the study and the novelty of the teaching method suggests that there is scope for expansion to cover more topics across a variety of specialties.

Furthermore, VR may provide an effective and interactive method of socially distanced learning in light of the COVID-19 pandemic.

\section{Conflicts of interest}

None declared. 